\title{
Enfermedad cerebrovascular de la circulación posterior: la importancia clínica
}

\author{
Irán Arce-Chavez* y Jesús E. Betancourt-Vera \\ Departamento de Urgencias, Unidad de Quemados, Hospital General de Zona N. ${ }^{\circ} 14$, Instituto Mexicano del Seguro Social, Hermosillo, Son., México
}

\begin{abstract}
Resumen
La enfermedad vascular cerebral consiste en subtipos divergentes que solo tienen en común el daño vascular al cerebro y se clasifica en dos grandes tipos: la isquemia (87\%) y la hemorragia (13\%). Aproximadamente 6.6 millones de estadounidenses han tenido un evento vascular cerebral y se estima que esta prevalencia se incrementará para el año 2030 en un 20.5\%. En México, el estudio BASID (Brain Attack Surveillance in Durango) reporta que la tasa anual se incrementa en forma significativa con la edad en todos los tipos. Una quinta parte de los infartos cerebrales son del territorio de la circulación posterior. La isquemia de la circulación posterior presenta desafíos en el diagnóstico y tratamiento. La enfermedad ateroesclerótica es su principal etiología. Los accidentes cerebrovasculares posteriores presentan gran variedad de síntomas y signos debido a la proximidad de los núcleos del tronco encefálico y a los grandes tractos aferentes y eferentes. La escala ROSIER (Recognition of Stroke In the Emergency Room) y la resonancia magnética son de vital importancia para el diagnóstico. El activador tisular del plasminógeno es una terapia eficaz para la enfermedad cerebrovascular de la circulación anterior y posterior, ofrece mejora en un 30\% de resultado funcional. El síndrome de Wallenberg tiene un pronóstico benigno, la supervivencia a los cinco años es del 54-59\%.
\end{abstract}

Palabras clave: Vascular cerebral. Isquemia. Circulación posterior. Wallenberg.

\section{Cerebrovascular disease of the posterior circulation: the clinical importance}

\begin{abstract}
He brain vascular disease consists of different subtypes that produce vascular damage to the brain in common and is classified into two major types: ischemia (87\%) and hemorrhage (13\%). Approximately 6.6 million Americans have suffered a ce $\neg$ rebrovascular event, and this prevalence is projected to increase by 2030 by $20.5 \%$. In Mexico, the BASID study reports that the annual rate increases significantly with age in all types. Roughly $50 \%$ of the cerebral infarctions are from the territo$r y$ of the poste-rior circulation. The posterior circulation ischemic treatment and diagnosis can be challenging. Atherosclerotic disease comprises the primary cause. Posterior circulation strokes have a variety of signs and symptoms that are close to the brainstem and the large afferent and efferent tracts. To evaluate the possible diagnosis in this study, ROSIER Scale and Magnetic resonance imaging (MRI) are being used. The tissue plasminogen activator (tPA) used as a therapy of the cerebrovascular disease of the anterior and posterior circulation showed an improvement of $30 \%$ of functional results. Wallenberg syndrome has a benign prognosis, the 5-year survival is $54-59 \%$.
\end{abstract}

Key words: Cerebral vascular. Ischemia. Posterior circulation. Wallenberg.

\footnotetext{
Correspondencia:

*Irán Arce-Chávez

E-mail: iarcechavez07@gmail.com

Fecha de recepción: 16-10-2019

DOI: 10.24875/REIE.19000069

Disponible en internet: 30-07-2021

Rev Educ Investig Emer. 2021;3(2):59-65 www.medicinadeemergencias.com

2604-6520 @ 2021 Sociedad Mexicana de Medicina de Emergencias, AC. Publicado por Permanyer México SA de CV. Este es un artículo open access bajo la licencia CC BY-NC-ND (http://creativecommons.org/licenses/by-nc-nd/4.0/).
} 


\section{Introducción}

Una quinta parte de los infartos cerebrales son del territorio de la circulación posterior y a pesar de la importancia de los eventos isquémicos de la circulación posterior reciben menos atención que aquellos de la circulación anterior. La isquemia de la circulación posterior presenta varios desafíos particulares en el diagnóstico y el tratamiento'. La enfermedad vascular cerebral consiste en subtipos divergentes que solo tienen en común el daño vascular al cerebro y se clasifica en dos grandes tipos: la isquemia (87\%) y la hemorragia (13\%). Aproximadamente 6.6 millones de estadounidenses han tenido un evento vascular cerebral y se estima que esta prevalencia se incrementará para el año 2030 en un 20.5\%. En México, el estudio BASID (Brain Attack Surveillance in Durango) reporta que la tasa anual se incrementa en forma significativa con la edad en todos los tipos ${ }^{2}$. El infarto medular lateral (síndrome de Wallenberg) se encuentra relacionado con la oclusión de la arteria vertebral intracraneal, en la que se altera el flujo sanguíneo a través de las perforantes hacia la médula lateral. Los infartos de la arteria cerebelar inferior posterior (ACPI) generalmente causan síndrome vertiginoso que se asemejan a la vestibulopatía periférica ${ }^{3}$.

\section{Anatomía vascular cerebral posterior}

El cerebro recibe sangre arterial de la arteria carótida interna ( $\mathrm{ACl}$ ) y la arteria vertebral (AV). La ACl transporta sangre a la circulación cerebral anterior y media; la AV transporta a la circulación posterior, incluida la arteria basilar (AB) y las arterias cerebrales posteriores (ACP). La circulación anterior y posterior se comunican formando el polígono de Willis. Sin embargo, el polígono de Willis solo se ve en hasta el $24 \%$ de las personas. La AV suministra la circulación posterior del tronco encefálico, los hemisferios cerebelosos y los hemisferios cerebrales posteriores. Esta circulación surge de las arterias subclavias. Por encima del nivel C1 la AV pasa a través de la duramadre en el agujero magno y da lugar a la ACPI, que son las primeras ramas intradurales de la $A V$, antes de anastomosarse en la línea media y dar lugar a la $A B$, la cual suministra a la arteria cerebelar inferior anterior, las arterias cerebelosas superiores, las arterias pontinas y finalmente la $\mathrm{ACP}^{4}$ (Fig. 1).

La ACPI proporciona sangre a la región dorsolateral de la médula y a una región de la superficie ventral de los hemisferios cerebelosos que incluyen el vermis inferior. Las arterias pontinas se dividen en dos clases: la primera, denominada paramediana, suministra a estructuras ubicadas cerca de la línea media, como el tracto corticoespinal; la segunda clase se llama circunflejo, por su distribución lateral que extiende sus ramas hacia la cara dorsal de la protuberancia. La arteria cerebelar inferior anterior suministra sangre a la superficie petrosa del cerebelo, también a la subregión del tronco encefálico pontino que incluye el pedúnculo cerebeloso medio y las porciones centrales de las vías sensoriales, y además da lugar a la arteria laberíntica del oído interno. La arteria cerebelosa superior (ACS) suministra al propio pedúnculo y la superficie superior del cerebelo, los núcleos profundos dentro de la materia blanca cerebelosa y una región del tallo cerebral ${ }^{5}$.

\section{Etiología}

La enfermedad ateroesclerótica afecta más frecuentemente al territorio de la ACPI, la AB, la ACS y ramas de la APC. En presencia de estenosis, se produce vasodilatación refleja debido a la circulación colateral inadecuada para aumentar el flujo sanguíneo cerebral y preservar el flujo normal, y la fracción de extracción de oxígeno aumentará a medida que el flujo se deteriore. Las enfermedades cardiacas con riesgo de embolia incluyen válvulas protésicas mecánicas, fibrilación auricular, trombo auricular o ventricular izquierdo, infarto de miocardio dentro de las primeras cuatro semanas posteriores al accidente cerebrovascular, miocardiopatía dilatada, endocarditis infecciosa, estenosis mitral e insuficiencia cardiaca congestiva. Las disecciones de la AV se presentan mayormente en pacientes jóvenes (1549 años), estos se presentan con isquemia y accidentes cerebrovasculares de la ACPI ${ }^{6}$.

\section{Fisiopatología}

El cerebro depende de la perfusión constante para el suministro de oxígeno y glucosa, cualquier interrupción del flujo aumenta el riesgo de infarto cerebral. La autorregulación cerebral puede estar condicionada por la musculatura vascular cerebral, que se contrae en respuesta de presiones más altas y se dilata en ausencia de presión, modulando así la resistencia para preservar una velocidad de flujo constante. Además, la disminución del flujo inicia la producción de sustancias vasodilatadoras que disminuyen la resistencia y aumentan el flujo ${ }^{7}$. Sin importar el evento precipitante, el resultado de la isquemia prolongada es la muerte celular cerebral. La cantidad suficiente para satisfacer las importantes demandas de energía del tejido cerebral 


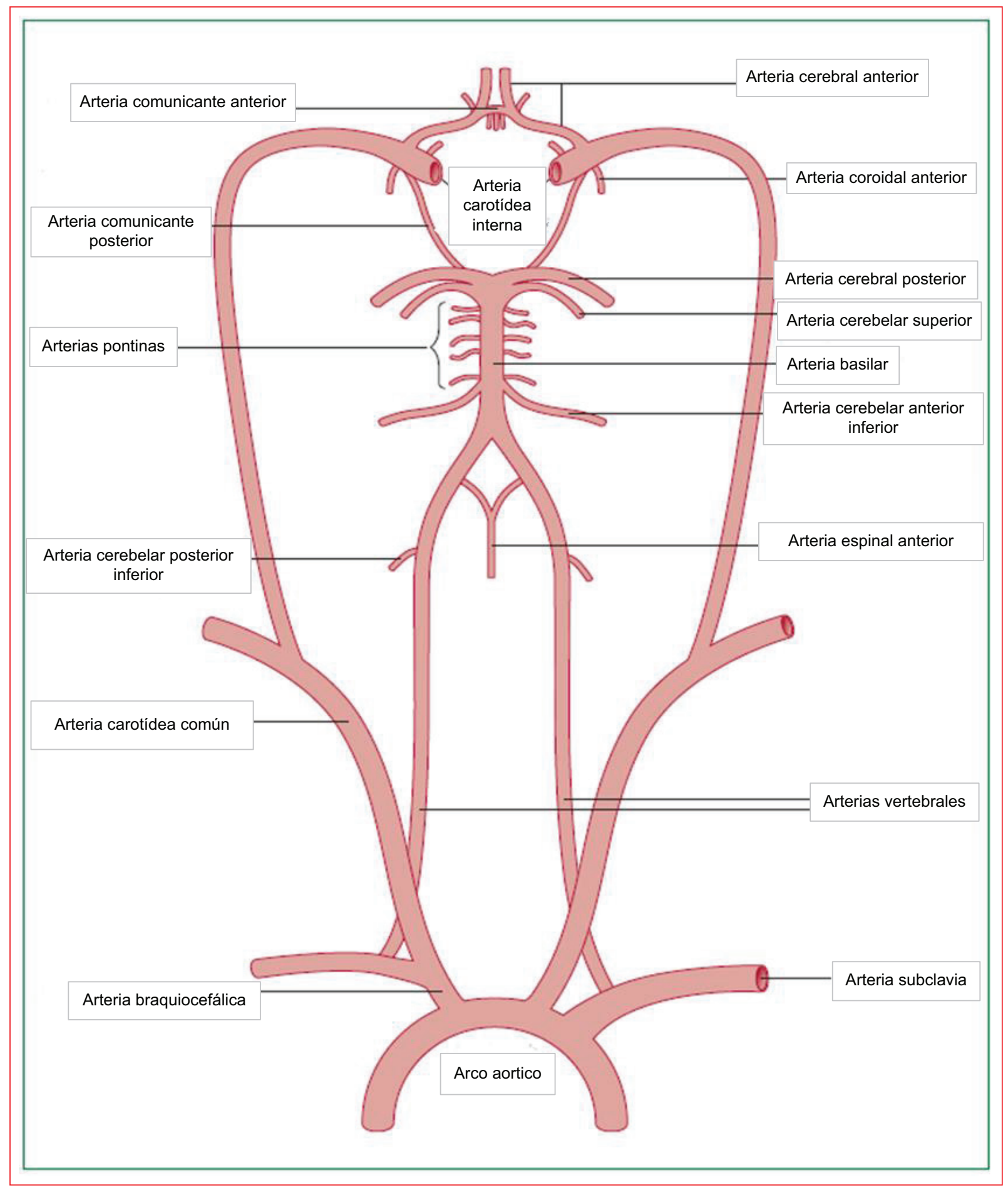

Figura 1. Anatomía cerebral arterial anterior y posterior (adaptada de Markus, et al., 2013¹).

es de $60 \mathrm{ml} / 100 \mathrm{~g} / \mathrm{min}$. Si el tejido experimenta una perfusión menor de $10 \mathrm{ml} / 100 \mathrm{~g} / \mathrm{min}$, esto condiciona falla de la membrana celular que produce daño cerebral de un grado de severidad e irreversibilidad que es proporcional a la duración de la isquemia ${ }^{8}$.

\section{Clínica}

Los accidentes cerebrovasculares posteriores suelen presentarse con una gran variedad de síntomas y signos debido a la proximidad de los núcleos del tronco 
Tabla 1. Síndrome lateral medular: presentación clínica y estructuras afectadas

\begin{tabular}{|c|c|}
\hline Estructura afectada & Síntomas y signos \\
\hline $\begin{array}{l}\text { Cerebelar y vestibular } \\
\text { - Núcleo vestibular } \\
\text { - Péndulo cerebelar } \\
\text { inferior }\end{array}$ & $\begin{array}{l}\text { - Mareos y vértigo } \\
\text { - Ataxia troncal, ataxia de extremidad } \\
\text { ipsilateral } \\
\text { - Hipotonía de brazo ipsilateral } \\
\text { - Visión borrosa, diplopía, oscilopsia } \\
\text { - Nistagmo multidireccional, } \\
\text { inclinación ocular (desviación } \\
\text { oblicua, posición ipsilateral del ojo } \\
\text { hacia abajo) }\end{array}$ \\
\hline $\begin{array}{l}\text { Sensorial } \\
\text { - Tracto } \\
\text { espinotalámico } \\
\text { medular lateral } \\
\text { - Núcleo trigémino } \\
\text { espinal y tracto } \\
\text { descendente }\end{array}$ & $\begin{array}{l}\text { - Pérdida de la percepción del dolor y } \\
\text { la temperatura en la cara ipsilateral, } \\
\text { el tronco y las extremidades } \\
\text { contralaterales } \\
\text { - Dolor y molestias faciales } \\
\text { ipsilaterales }\end{array}$ \\
\hline $\begin{array}{l}\text { Bulbar } \\
\text { - Núcleo ambiguo }\end{array}$ & $\begin{array}{l}\text { - Ronquera y disfagia } \\
\text { - Debilidad del paladar ipsilateral, } \\
\text { faringe y laringe } \\
\text { - Hipo }\end{array}$ \\
\hline $\begin{array}{l}\text { Autonómico } \\
\text { - Tegmento lateral } \\
\text { - Sistema nervioso } \\
\text { simpático } \\
\text { descendente }\end{array}$ & $\begin{array}{l}\text { - Fracaso de la respiración } \\
\text { automática, particularmente durante } \\
\text { el sueño } \\
\text { - Síndrome de Horner ipsilateral }\end{array}$ \\
\hline
\end{tabular}

Adaptada de Shultz, et al., $2016^{9}$.

encefálico y a los grandes tractos aferentes y eferentes ${ }^{9}$. El vértigo y los mareos son síntomas muy comunes en el infarto de la circulación posterior y pueden exacerbarse con el movimiento de la cabeza, estos están presentes en el $47-75 \%$ de los casos. El nistagmo esta presente el $24-48 \%$ de los infartos de la circulación posterior ${ }^{10}$. En un estudio realizado por Park, et al. documentaron que los pacientes que presentaban lesión en la región dorsolateral de la médula media presentaban singulto (14\%, 7 pacientes de 55$)^{11}$. El síndrome más común relacionado con la oclusión de la ACPI es el síndrome lateral medular (síndrome de Wallenberg). La característica más predominante del infarto de la ACPI es el vértigo. La ataxia troncal es otra característica destacada del infarto de la ACPI, la presentación clínica de este síndrome está resumida en la tabla 1. La presentación clínica de la $A B$ es variable, depende de la etiología, anatomía vascular y la presencia de circulación lateral. Las características clínicas se resumen en la tabla 2. La ACP contiene una clínica variable, los defectos del campo visual son la característica más común, los déficits de la memoria y varios síndromes de desconexión que afectan las vías

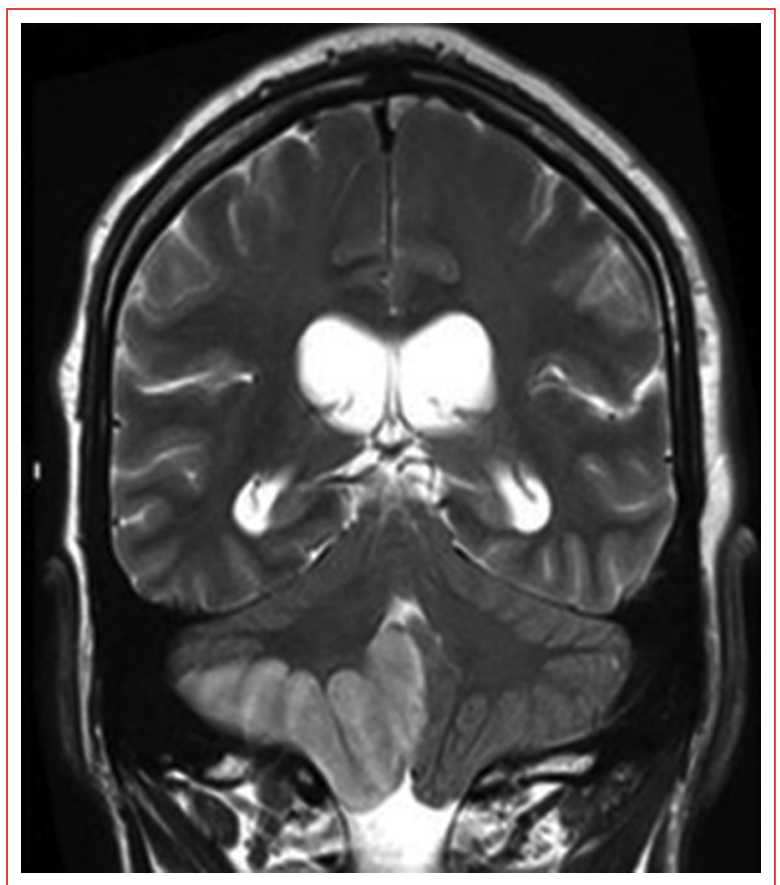

Figura 2. Secuencia de Resonancia Magnética T2 muestra infarto cerebeloso derecho con edema y efecto de masa (adaptada de Nouh, et al., 20146).

visuales y del lenguaje, por ejemplo: alexia sin agrafia, síndrome de Gerstmann y prosopagnosia, otro síntoma común es la cefalea, que puede confundirse con la migraña, especialmente si hay síntomas visuales ${ }^{9}$.

\section{Diagnóstico}

El diagnóstico del accidente cerebrovascular de la circulación posterior se basa en la historia y el examen clínico con apoyo de imágenes. Existen herramientas diagnósticas para el personal médico en el departamento de urgencias ${ }^{3}$. En la sala de urgencias se cuenta con una herramienta para el diagnóstico del accidente cerebrovascular de la circulación posterior: la escala ROSIER (Recognition of Stroke In the Emergency Room), la cual evalúa los defectos visuales con una sensibilidad del $93 \%$ y una especificidad del $83 \%^{12}$. Aunque todavía le da más peso al diagnóstico la escala NIHSS (National Institutes of Healt Stroke Scale), al incluir síntomas específicos del accidente cerebrovascular de la circulación posterior como la ataxia de la marcha y la pérdida del campo visual ${ }^{13}$. La prueba de impulso de cabeza, evaluación de nistagmo y prueba de desviación de inclinación (HINTS, por sus siglas en inglés) (Tabla 3) es un examen dinámico que prueba el reflejo vestibuloocular. Ayuda a la diferenciación del vértigo de causa central o 
Tabla 2. Infarto de la arteria basilar y sus ramas: presentación clínica y estructuras afectadas

\begin{tabular}{|c|c|c|}
\hline Vaso afectado & Estructura afectada & Presentación clínica \\
\hline $\begin{array}{l}\text { Arteria basilar inferior y media } \\
\text { - Perforantes paramedios pontinas, } \\
\text { ramas circunferenciales cortas }\end{array}$ & $\begin{array}{l}\text { - Tracto corticoespinal } \\
\text { - Tracto corticobulbar } \\
\text { - Formación reticular pontina } \\
\text { paramediana } \\
\text { - Lemnisco medial }\end{array}$ & $\begin{array}{l}\text { - Debilidad unilateral o bilateral de extremidades } \\
\text { - Síntomas bulbares: disartria, disfonía, disfagia } \\
\text { - Anormalidades del movimiento ocular: parálisis de la } \\
\text { mirada horizontal, oftalmoplejía internuclear, nistagmo, } \\
\text { pupilas puntiformes } \\
\text { - Déficits sensoriales }\end{array}$ \\
\hline $\begin{array}{l}\text { Parte superior de la arteria basilar } \\
\text { - Ramas perforantes del } \\
\text { mesencéfalo } \\
\text { - Arteria cerebelosa superior (ver } \\
\text { arteria cerebelosa anterior inferior) } \\
\text { - Arteria cerebral posterior }\end{array}$ & $\begin{array}{l}\text { - Mesencéfalo/región pretectal } \\
\text { - Sistema de activación reticular } \\
\text { - Tálamo } \\
\text { - Lóbulo temporal medial } \\
\text { - Corteza occipital }\end{array}$ & $\begin{array}{l}\text { - Anormalidades del movimiento ocular vertical } \\
\text { - Hiperconvergencia } \\
\text { - Hipersomnolencia } \\
\text { - Alucinaciones pedunculares } \\
\text { - Déficits sensoriales } \\
\text { - Amnesia anterógrada y retrógrada } \\
\text { - Defectos del campo visual }\end{array}$ \\
\hline Arteria cerebelosa anterior inferior & $\begin{array}{l}\text { - Mesencéfalo lateral } \\
\text { - Cerebelo superior }\end{array}$ & $\begin{array}{l}\text { - Parálisis del IV par craneal, pérdida sensorial } \\
\text { hemifacial ipsilateral, pérdida hemisensorial del cuerpo } \\
\text { contralateral, síndrome de Horner, ataxia de } \\
\text { extremidades, dismetría } \\
\text { - Ataxia troncal y de la marcha, disartria, náuseas y } \\
\text { vómitos (pseudogastroenteritis) }\end{array}$ \\
\hline $\begin{array}{l}\text { Síndrome de enclaustramiento } \\
\text { - Arterias perforantes pontinas, } \\
\text { escaso suministro colateral }\end{array}$ & - Infarto pontino extenso & $\begin{array}{l}\text { - Pérdida de todas las extremidades y la función motora } \\
\text { bulbar. Solo se mantienen los movimientos oculares } \\
\text { verticales. Conciencia y cognición preservadas }\end{array}$ \\
\hline
\end{tabular}

Adaptada de Shultz, et al., $2016^{9}$.

Tabla 3. Prueba de impulso de cabeza, evaluación de nistagmo y prueba de desviación de inclinación (HINTS)

Impulso de la cabeza - Nistagmo - Prueba de inclinación (HINTS)

Prueba de impulso de cabeza horizontal (h-HIT)

- El sujeto fija los ojos en un objetivo central. Gira la cabeza de forma rápida e impredecible de lado a lado unos $15^{\circ}$

- Respuesta normal: los ojos permanecen fijos en el objetivo (h-HIT negativo)

- Respuesta anormal: movimiento ocular correctivo al girar la cabeza hacia el lado del daño vestibular periférico. Debido a la falta de entrada vestibular, no se puede mantener la posición del ojo

- Una h-HIT negativa en un paciente con síntomas vestibulares sugiere una lesión central

- Puede ocurrir una h-HIT positiva en pacientes con infarto de pontina lateral, lo que hace que la prueba sea menos específica para lesiones vestibulares periféricas

Nistagmo

- Lesiones periféricas: el nistagmo unidireccional, predominantemente horizontal, aumenta de intensidad cuando el paciente mira en la dirección de la fase rápida del nistagmo

- Lesiones centrales: nistagmo vertical o torsional. El nistagmo horizontal en una lesión central puede ser indistinguible del nistagmo que ocurre en las lesiones periféricas, aunque puede cambiar de dirección con un cambio en la dirección de la mirada.

Desviación oblicua

- Predictivo de lesión central (rara vez puede ocurrir en lesiones periféricas)

- Test de prueba de cobertura alternativa: con desviación de inclinación, el ojo se desplaza verticalmente cuando se descubre

Lesión central

- $\mathrm{h}$-HIT negativo bilateralmente

- Nistagmo multidireccional

- Desviación oblicua presente

- Artículos presentes o no comprobables
Lesión periférica

- Positivo h-HIT

- Nistagmo horizontal unidireccional, que aumenta en intensidad con la dirección de la mirada hacia la fase rápida

- Desviación oblicua ausente

- Todos los hallazgos presentes 


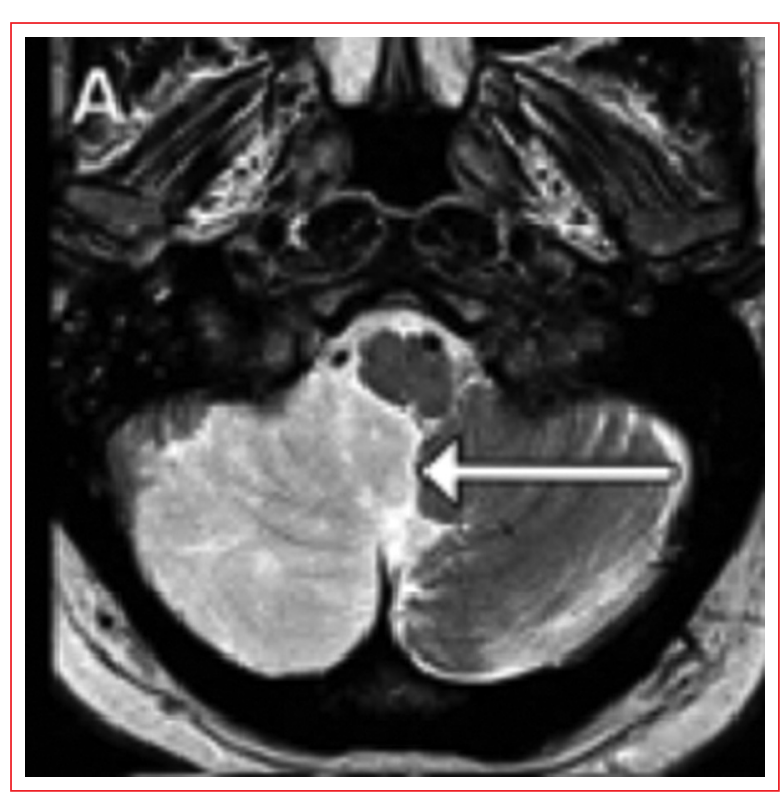

Figura 3. Hallazgo de imagen asociado con infarto de la ACPI derecha (flecha) (adaptada de Merwick, et al., 2014 ).

periférica ${ }^{10}$. La tomografía simple de cráneo es un estudio indispensable para detectar hemorragia intracraneal - lesiones tumorales que son contraindicaciones de recibir tratamiento trombolítico, pero tienen muy baja sensibilidad para detectar infartos hiperagudos 0 agudos de la circulación posterior. En la figura 2 y figura 3 podemos observar algunas imágenes de resonancia magnética de encéfalo como el estudio de preferencia para excluir el infarto cuando se sospecha de accidente cerebrovascular de la circulación posterior ${ }^{14}$.

\section{Tratamiento}

El activador tisular del plasminógeno (t-PA) es una terapia eficaz para la enfermedad cerebrovascular de la circulación posterior que ofrece mejora en un $30 \%$ de resultado funcional del accidente cerebrovascular. Sin embargo, los síntomas de la circulación posterior son comunes e inespecíficos, y a menudo son una manifestación de una etiología no neurológica, lo cual dificulta la sospecha diagnóstica. Si bien los retrasos prehospitalarios son una barrera importante, el reconocimiento de los síntomas en la sala de urgencias pueden provocar un retraso en el diagnóstico, reducir la elegibilidad para el tratamiento con t-PA o retrasar el tratamiento ${ }^{15}$. No se ha encontrado diferencias en cuanto al tratamiento con t-PA dentro de las primeras 4.5 horas de inicio de los síntomas entre enfermedad cerebrovascular de la circulación anterior y la posterior.

Existen pocos estudios en los que se ha ofrecido el tratamiento con t-PA para manejo del infarto de la ACPI a dosis de $0.9 \mathrm{mg} / \mathrm{kg}$ de peso (máximo $90 \mathrm{mg}$ ) con el uso del $10 \%$ en bolo y el resto para 60 minutos, tal es el caso del estudio IST-3 (Third International Stroke Trial), que buscó determinar si un rango más amplio de pacientes podría beneficiarse hasta seis horas del inicio del accidente cerebrovascular; de 3,035 pacientes, 246 presentaron infarto de la ACPI, consiguiendo una reperfusión del $60 \%$ en el grupo del t-PA vs. $58.1 \%$ del grupo control ${ }^{16}$. Existe en la literatura el reporte de caso de un paciente con estenosis de la $A B$ tratado tres días después con dosis bajas de administración intravenosa de t-PA a 0.125 $\mathrm{mg} / \mathrm{kg}$, que se infundió continuamente durante 48 horas, concluyendo en un resultado clínico favorable ${ }^{17}$.

En un estudio dirigido por Hamenter y publicado en Neurocritical Care se realiza fibrinólisis prolongada con t-PA en pacientes con trombosis de la $A B$ a dosis de $5.24 \mathrm{mcg} / \mathrm{kg} / \mathrm{h}$ (no más de $10 \mathrm{mg}$ en 24), con resultados favorables para 9 de 14 pacientes $^{18}$.

La trombectomía mecánica con extracción asistida por vacío recuperada con stent es una opción terapéutica en centros con terapia endovascular neurológica. Maus, et al. analizaron retrospectivamente a 66 pacientes con trombectomía mecánica para oclusión de grandes vasos de la circulación posterior. Entre el 61 y el $67 \%$ de los tratados con extracción asistida por vacío recuperada con stent obtuvieron reperfusión exitosa ${ }^{19}$.

\section{Complicaciones}

El síndrome de Wallenberg tiene un pronóstico benigno, la supervivencia a los cinco años es del $54-59 \%$; el $40 \%$ de los fallecimientos es por un nuevo infarto cerebral y el $26 \%$ por un infarto de miocardio ${ }^{20,21}$. Los trastornos de hipoventilación central surgen de la integración inadecuada de la señal del centro de impulsión respiratoria central en el tronco encefálico inferior, que puede deberse a una disfunción adquirida o congénita; generalmente ocurre debido a lesiones traumáticas, isquémicas o inflamatorias del tronco encefálico. El síndrome de hipoventilación central secundario, también conocido como la maldición de Ondine, es una complicación del síndrome de Wallenberg que se caracteriza por la falla de los mecanismos respiratorios mientras se duerme, los pacientes presentan apnea persistente durante el sueño ${ }^{22}$.

Otras posibles complicaciones del accidente cerebrovascular vertebrobasilar incluyen: neumonía por aspiración, trombosis venosa profunda y embolia pulmonar. Una 
revisión de la literatura realizada por Yuan, et al. indicó que el factor de riesgo para la infección pulmonar en pacientes con accidente cerebrovascular es haber presentado múltiples accidentes cerebrovasculares, así como otros factores: NIHSS de más de 15 puntos, uso de ventilación mecánica, uso de sondas nasogástricas y disfagia ${ }^{23}$.

Los pacientes que reciben manejo con t-PA presentan riesgo de sangrado. La hemorragia intracerebral sintomática dentro de las 36 horas posteriores a la aparición del accidente cerebrovascular ocurrió en el $6.4 \%$ de los pacientes que recibieron t-PA ${ }^{24}$; se ha demostrado que del $1-4 \%$ presentan microhemorragias cerebrales clínicamente silenciosos ${ }^{25}$.

\section{Conclusión}

La enfermedad cerebrovascular de la circulación posterior es un síndrome clínico de difícil diagnóstico. Sus características clínicas son variables, pero tienen una relación estrecha con los diferentes sitios de oclusión. Además, durante la evaluación del paciente con sospecha de oclusión de la circulación posterior se deben implementar todas las herramientas disponibles para diferenciar de otros síndromes que asemejan la misma presentación clínica. La arteria con mayor frecuencia de oclusión es la ACPI, siendo esta la primera rama de la circulación intracraneal posterior. Los estudios de imagen se deben obtener en breve tras el ingreso; la resonancia magnética es el estudio de preferencia para el diagnóstico de la fosa posterior. A pesar de no haber diferencias en el tratamiento farmacológico de la circulación anterior y la posterior, este no se debe de retrasar, con el fin de disminuir el mal pronóstico y las secuelas permanentes.

\section{Financiamiento}

La presente investigación no ha recibido ninguna beca específica de los sectores público, comercial o sin ánimo de lucro.

\section{Conflicto de intereses}

Los autores declaran no tener conflicto de intereses.

\section{Responsabilidades éticas}

Protección de personas y animales. Los autores declaran que para esta investigación no se han realizado experimentos en seres humanos ni en animales.

Confidencialidad de los datos. Los autores declaran que en este artículo no aparecen datos de pacientes.
Derecho a la privacidad y consentimiento informado. Los autores declaran que en este artículo no aparecen datos de pacientes.

\section{Bibliografía}

1. Markus HS, van der Worp B, Rothwell PM. Posterior circulation ischaemic stroke and transient ischaemic attack: diagnosis, investigation, and secondary prevention. Lancet Neurol. 2013;12(10):989-98.

2. Diagnóstico y tratamiento temprano de la enfermedad vascular cerebral isquémica en el segundo y tercer nivel de atención [Internet]. Ciudad de México: Secretaría de Salud; 09/03/2017. Disponible en: http://www.cenetec.salud.gob.mx/contenidos/gpc/catalogoMaestroGPC.html

3. Merwick A, Werring D. Posterior circulation ischaemic stroke. BMJ. 2014;348:g3175.

4. Prince EA, Ahn SH. Basic vascular neuroanatomy of the brain and spine: what the general interventional radiologist needs to know. Semin Intervent Radiol. 2013;30(3):234-9.

5. Chandra A, A Li W, Stone CR, Geng X, Ding Y. The cerebral circulation and cerebrovascular disease: anatomy. Brain Circ. 2017;3(2):45-56.

6. Nouh A, Remke J, Ruland S. Ischaemic posterior circulation stroke: a review of anatomy, clinical presentations, diagnosis and current management. Front Neurol. 2014;5(30):1-16.

7. Chandra A, Stone CR, A Li W, Geng X, Ding Y. The cerebral circulation and cerebrovascular disease II: Pathogenesis of cerebrovascular disease. Brain Circ. 2017;3(2):57-65.

8. Chandra A, Stone CR, A Li W, Geng X, Ding Y. The cerebral circulation and cerebrovascular disease III: Stroke. Brain Circ. 2017;3(2):66-77.

9. Shultz UG, Fisher U. Posterior circulation cerebrovascular syndromes: diagnosis and management. Neurol Neurosurg Psychiatry. 2016;88(1):45-53.

10. Venhovens J, Meulstee J, Verhagen WIM. Acute vestibular syndrome: a critical review and diagnostic algorithm concerning the clinical differentiation of peripheral versus central aetiologies in the emergency department. J Neurol. 2016;263(11):2151-7.

11. Park MH, Kim BJ, Koh SB, Park MK, Lee DH. Lesional location of lateral medullary infraction presenting hiccups (singultus). J Neurol Neurosurg Psychiatry. 2005;76(1):95-8.

12. Nor AM, Davis J, Sen B, Shipsey D, Louw S, Dyker AG, et al. The recognition of stroke in the emergency room (ROSIER) scale: development and validation of a stroke recognition instrument. Lancet Neurol. 2005;4(11):727-34.

13. Dornák $T$, Král M, Šanák $D$, Kanovský $P$. Intravenous thrombolysis in posterior circulation stroke. Front Neurol. 2019;10:417.

14. Hwang DY, Silva GS, Furie KL, Greer DM. Comparative sensitive of computed tomography vs. magnetic resonance imaging for detecting acute posterior fossa infarct. J Emerg Med. 2012;24(5):559-65.

15. Sarraj A, Medrek S, Albright K, Martin-Schild S, Bibars W, Vahidy F, et al. Posterior circulation stroke is associated with prolonged door to needle time. 2015;10(5):672-8.

16. IST-3 Collaborative Group, Sandercock P, Wardlaw JM, Lindley RI, Dennis M, Cohen G, et al. The benefits and harms of intravenous thrombolysis with recombinant tissue plasminogen activator within $6 \mathrm{~h}$ of acute ischaemic stroke (the third international stroke trial [IST-3]): a randomised controlled trial. Lancet. 2012;379(9834):2352-63.

17. Dornak, T, Herzig R, Sanak, D, Skoloudik, D. Management of acute basilar artery occlusion: Should any treatment strategy prevail?. Biomed Pap Med Fac Univ Palacky Olomouc Czech Repub. 2014;158(4):528-34.

18. Hametner C, Kellert L, Veltkamp R, Behrens L, Nagel S, Hacke W, et al. Prolonged low-dose thrombolysis in posterior circulation stroke. Neurocrit Care. 2013;21(1):114-8.

19. Maus V, Stycze H, Liman J, Maier I, Brehm A, Tsogkas I, et al. Intracranial mechanical thrombectomy of large vessel occlusions in the posterior circulation using SAVE. BMC Neurol. 2019;19(197):1-6.

20. Roldán-Valdez E, Juárez-Jiménez H, Corona-Cedillo R, Martínez-López M. Síndrome de Wallenberg: Hallazgos en resonancia magnética con correlación clínica. Gac Méd Méx. 2007;143(5):429-32.

21. Sacco RL, Freddo L, Bello JA, Odel JG, Onesti ST, Mohr JP. Wallenberg's lateral medullary syndrome. Clinical-magnetic resonance imaging correlations. Arch Neurol .1993;50(6):609-14.

22. Sivacumar K, Garcha M, Mehta D, Leary MC, Yacoub HA. Central hipoventilation: a rare complication of Wallenberg syndrome. Case Rep Neurol Med. 2018;2018:1-3.

23. Yuan MZ, Li F, Tian X, Wang W, Jia M, Wang XF, et al. Risk factors for lung infection in stroke patients: a meta-analysis of observational studies. Expert Rev Anti Infect Ther. 2015;13(10):1289-98.

24. Marler J. Tissue plasminogen activator for acute ischemic stroke. N Engl J Med. 1995;333(24):1581-8.

25. Powers WJ, Rabinstein AA, Ackerson T, Adeoye OM, Bambakidis NC, Becker K, et al. 2018 Guidelines for Early Management of Patients With Acute Ischemic Stroke: A guideline for healthcare professionals from the American Heart Association/American Stroke Association. Stroke. 2018;49(3):e46-e99. 